\title{
Establishment and characterization of a novel human osteosarcoma cell line for spontaneous pulmonary metastasis research in vivo
}

\author{
Pei Yu ${ }^{1 \#}$, Junxiang Wen ${ }^{1 \#}$, Jun Wang ${ }^{2}$, Jing Liang ${ }^{2}$, Yuhui Shen ${ }^{1}$, Weibin Zhang ${ }^{1}$ \\ ${ }^{1}$ Department of Orthopedics, Ruijin Hospital Affiliated to Shanghai Jiao Tong University School of Medicine, Shanghai 200025, China; ${ }^{2}$ Shanghai \\ Institute of Traumatology and Orthopedics, Shanghai 200025, China \\ Contributions: (I) Conception and design: W Zhang; (II) Administrative support: W Zhang; (III) Provision of study materials or patients: Y Shen; (IV) \\ Collection and assembly of data: P Yu, J Wen; (V) Data analysis and interpretation: P Yu, J Wen; (VI) Manuscript writing: All authors; (VII) Final \\ approval of manuscript: All authors. \\ "These authors contributed equally to the work. \\ Correspondence to: Yuhui Shen; Weibin Zhang. Department of Orthopedics, Ruijin Hospital Affiliated to Shanghai Jiao Tong University School of \\ Medicine, Shanghai 200025, China. Email: yuhuiss@163.com; zhangweibin10368@163.com.
}

Background: A large number of osteosarcoma patients are dying of pulmonary metastasis in spite of the
recent progress achieved in treatment research. Therefore, there is an urgent need for an in vivo experiment,
whose purpose is to investigate the growth and metastasis of human osteosarcoma tumor. In this study, a
novel human osteosarcoma cell line was established and characterized for osteosarcoma metastatic research
in vivo.

Methods: Small pieces of parental primary osteosarcoma samples from a 16-year-old boy were xenotransplanted into NOD/SCID mice. Then, osteosarcoma cells from the xeno-grafts were isolated and further passaged in vitro. Expression profiling was confirmed using HE staining and immunohistochemistry. The tumorigenic and metastatic ability of the established cell line was analyzed by cell scratch and CCK8 assay in vitro, and tumor transplantation in NOD/SCID mice.

Results: Parental cells were pleomorphic and positive with ALP, SSEA-4, and CD44. Osteosarcoma cells, named Well5 cells, were successfully isolated and had the ability of adipogenesis and osteogenesis. Well5 cells were mostly positive for SSEA-4 protein, and possessed morphological characteristics such as osteoblastic nature during the cultivation and hetero transplantation. Cell scratch and CCK8 assay indicated Well5 cells obtained a better capacity for migration and proliferation than the MG63 cell line. In NOD/ SCID mice, orthotopic tumors were established at the primary site, and spontaneous pulmonary metastases were developed.

Conclusions: A novel human osteosarcoma cell line was successfully established, which may be helpful for spontaneous pulmonary metastasis research in vivo.

Keywords: Human osteosarcoma; Well5 cell line; xeno-transplantation; spontaneous pulmonary metastasis

Submitted Aug 18, 2019. Accepted for publication Aug 27, 2019.

doi: 10.21037/atm.2019.09.23

View this article at: http://dx.doi.org/10.21037/atm.2019.09.23

\section{Introduction}

Osteosarcoma is the most common malignancy of bones in humans, which accounts for $\sim 2.4 \%$ of childhood tumors (1). The incidence peaks during the second decade of life, and the tumors mainly localize in the distal femur and proximal tibia $(2,3)$. For most local lesions, osteosarcoma can be surgically cured with a 5 -year survival of $~ 50 \%$ to $65 \%(4,5)$. Meanwhile, approximately $80 \%$ of patients have metastatic 
diseases $(6,7)$, especially pulmonary metastases, which accounts for the top cause of death $(8,9)$.

In spite of the advances achieved in the treatment, the mechanism underlying tumor progression and metastasis remains elusive. A transplanted-tumor animal model is necessary to clarify the mechanism underlying the progression and metastasis of osteosarcoma in vivo (10). A previous study reported the establishment and characterization of a model. In this study, human osteosarcoma cells were transformed with $\mathrm{v}$-Ki-ras, and were producible for pulmonary colonies by intravenous injection into nude mice (11). However, an animal model with a transplanted primary solid tumor that can develop spontaneous pulmonary metastasis has been less reported.

In this study, we developed a novel human osteosarcoma cell line that was suitable for establishing a transplanted primary solid tumor and developing a spontaneous pulmonary metastasis in animals.

\section{Methods}

\section{Human osteosarcoma xeno-transplantation}

Human primary osteosarcoma tissues were obtained from a 16-year-old boy with osteoblastic osteosarcoma, which was in accordance with the ethical guidelines established by Shanghai Ruijin Hospital. Fresh osteosarcoma tissues (named WJ0823, P0) were dissected into small pieces $\left(3 \times 3 \times 1 \mathrm{~mm}^{3}\right)$ at $4{ }^{\circ} \mathrm{C}$, and implanted subcutaneously into the two flanks of 6-to-8-week-old female NOD/SCID mice (P1) through a $3-\mathrm{mm}$ incision. Then, tumor tissues from the xeno-grafts were dissected into small pieces $\left(2 \times 2 \times 1 \mathrm{~mm}^{3}\right)$ and implanted into the 2 flanks of another NOD/SCID mouse (P2) for further passage. All animal experiments were in accordance with the approved guidelines provided by the Laboratory Animal Resource Center of Shanghai Jiao Tong University School of Medicine.

\section{Human osteosarcoma protein marker detected by bematoxylin and eosin (HE) staining and immunobistochemistry}

This patient's osteosarcoma tissues including $\mathrm{P} 0, \mathrm{P} 1$, and $\mathrm{P} 2$ tissues were fixed in $10 \%$ buffered formalin and decalcified. All tissues were routinely embedded in paraffin and 3 nonsequential sections/animal were obtained. The tissues were subjected to Cal-Ex solution overnight. Then, all tissues were cut into $5 \mu \mathrm{m}$ sections and stained with hematoxylin and eosin according to the manufacturer's instruction.

These tissues were tested for alkaline phosphatase (ALP, Abcam, UK, 1:1,000), SSEA-4 (Santa Cruz, USA, 1:1,000), and CD44 (BD, USA, 1:1,000) expression by immunohistochemistry using a StreptAvidin-Biotinylated Peroxidase complex (ABC) kit (Boster, China) according to the manufacturer's instruction.

All the slides were examined using alight microscope (Olympus BX60).

\section{Preparation, purification, and characterization of Well5 cell suspensions from induced subcutaneous tumors at the flank of the mice}

The subcutaneous tumors (P2) at the flank of the mice were harvested 6 weeks after tumor inoculation, and a singlecell suspension was prepared by digesting tumor tissue $\left(4 \mathrm{~mm}^{3}\right)$ with type IV collagenase for $1 \mathrm{~h}$ at $37^{\circ} \mathrm{C}$. Then, cells were washed in PBS3 times, and grown in DMEM Medium (Life Technologies, USA) supplemented with $10 \%$ FBS (Life Technologies, USA) at $37{ }^{\circ} \mathrm{C}$ in a $5 \% \mathrm{CO}_{2}$ incubator. Flow cytometric analysis was used to distinguish between $\mathrm{CD}_{4} 4^{+}$human (BD, USA) cells and $\mathrm{CD} 44^{-}$mice cells. Cells were suspended in FACS buffer (PBS containing $1 \% \mathrm{FBS}$, with or without $0.1 \% \mathrm{Na} 3 \mathrm{~N}$ ) and subsequently stained with fluorochrome-conjugated antibodies. Then, data were analyzed with either FlowJo software (Tree Star, Ashland, OR, USA) or Summit software (Beckman coulter). Finally, CD $44^{+}$Cellswere sorted by MoFlo and suspended into DMEM supplemented with $2 \%$ FBS.

Sorted $\mathrm{CD} 44^{+}$cells were passaged two times in vitro, and then digested and diluted into 10 divisions. Each division was digested and diluted repeatedly until the purity of the diluted osteosarcoma cells was close to $100 \%$. Well5 cells were tested for SSEA-4 expression by immunofluorescence and SSEA-4 (Santa Cruz, CA, USA, 1:1,000), CD44 (BD, USA, 1:1,000), CD105 (BD, USA, 1:500), and CD166 (BD, USA, 1:500) expression by flow cytometry.

\section{Induced differentiation of Well5 cells in vitro}

Well5 cells were seeded at a density of 26,000 cells $/ \mathrm{cm}^{2}$ and cultured either in proliferation medium or inadipomaintenance medium [ $\alpha$-MEM supplemented with $10 \%$ FBS, $1 \%$ PSN, and $10 \mu \mathrm{g} / \mathrm{mL}$ insulin (Sigma, Cat\#I-6634)] for $24 \mathrm{~h}$ followed by cultivation in adipo-induction medium [ $\alpha$-MEM supplemented with $10 \%$ FBS, $1 \%$ PSN, $1 \mu M$ dexamethasone (Sigma, Cat\#D-4902), $10 \mu \mathrm{g} / \mathrm{mL}$ insulin, 
$0.5 \mathrm{mM}$ isobutyl-1-methylxanthine (Sigma, Cat\#I-5879), and $200 \mu \mathrm{M}$ indomethacin (Sigma, Cat\#I-7378)] for $72 \mathrm{~h}$.

To induce differentiation, Well5 cells were seeded at a density of 2,000 cells $/ \mathrm{cm}^{2}$ and cultured either in proliferation medium or in differentiation medium [ $\alpha$-MEM supplemented with 10\% FBS, 1\% PSN, $10 \mathrm{nM}$ dexamethasone, $50 \mathrm{mM}$ ascorbate-2-phosphate, $2 \mathrm{mM}$ $\beta$-glycerophosphate (Sigma, \#G-9891), and $10 \mathrm{nM}$ 1,25-dihydroxyvitamin D3 (Sigma, \#D-1530)]. The media were replaced every 3-4 days for up to 7 days.

\section{In vitro cell scratch and proliferation assay}

A scratch was made across the monolayer of Well5 cells seeded in 6-well plates. Well5 cells were seeded at a density of about $2.5 \times 10^{5}$ cells/well in complete medium (DMEM with $10 \%$ FBS). When the well was covered with Well5 cells, scratch was performed. Images of the scratches were captured at 0 and $24 \mathrm{~h}$ using inverted microscope (IX73, Olympus, Tokyo, Japan). CCK-8 assay (Dojindo, Rockville, MD, USA) was used to investigate cell proliferation. Briefly, $100 \mu \mathrm{L}$ of cell suspension (5,000 cells/well) were seeded in a 96well plate, and incubated in a humidified incubator at $37{ }^{\circ} \mathrm{C}$ for $24 \mathrm{~h}$. Then, $10 \mu \mathrm{L}$ of CCK-8 was added in each well, and incubated at $37^{\circ} \mathrm{C}$ for $2 \mathrm{~h}$. Finally, OD $450 \mathrm{~nm}$ was measured using a microplate reader.

\section{Ortbotopic intratibial injection and monitoring tumor growth and metastasis}

A total of $24 \mathrm{BALB} / \mathrm{c}$ nude mice were anesthetized with ketamine $(100 \mathrm{mg} / \mathrm{kg})$. Well5 cells $\left(1 \times 10^{6}\right.$ cells in $200 \mu \mathrm{L}$ PBS) were injected into the proximal tibia of the mice. The tumor size was measured every 3 days, and the primary tumors and pulmonary metastatic lesions were harvested at 4,6 , and 8 weeks after injection. The metastatic lesions were fixed in $10 \%$ formalin, and embedded in paraffin. Then, 3 non-sequential serial sections/animal were stained with $\mathrm{HE}$ staining form icroscopic metastasis analysis.

\section{In vivo imaging system (IVIS)}

Luciferin was injected into the intraperitoneal cavity $\sim 5$ minutes before imaging at a dose of $150 \mathrm{mg} / \mathrm{kg}$. Mice were then placed on the imaging stage to collect ventral and dorsal images using the IVIS (Xenogen Corp., Alameda, CA, USA). Photons emitted from the primary tumors and pulmonary metastatic lesions were quantified using Living
Image software (Xenogen Corp., Alameda, CA, USA).

\section{Statistical analysis}

SPSS19.0 software package was used for data analysis. Measurement data are presented as mean \pm SD of 3 independent experiments. Student's $t$-test for unpaired variables was used to determine statistical significance of differences between the groups, and a $\mathrm{P}<0.05$ was considered statistically significant.

\section{Results}

\section{Characterization of buman osteosarcoma tissues and tissue transplantation}

Firstly, we identified the WJ0823 human osteosarcoma tissues by immunohistochemistry staining, human osteosarcoma cells were pleomorphic and positive with alkaline phosphatase reaction, SSEA-4, and CD44 (Figure 1A). The genetic background of human osteosarcoma detected by fluorescence in situ hybridization (FISH) showed that retinoblastoma $(\mathrm{Rb})$ gene was deficient (data not shown). To identify the tumorigenicity of parent osteosarcoma tissue, we subcutaneously inoculated minced human osteosarcoma tissue into NOD/SCID mice; the primary specimens generated passage $1(\mathrm{P} 1)$ (Figure $1 B ; \mathrm{b} 1, \mathrm{~b} 3)$ and passage 2 (P2) xenografts (Figure $1 B$; b2,b4). In addition, in animals injected with human osteosarcoma tissues, the tumors caused a destruction of tibia by $\mathrm{X}$-ray images, including bone destruction of the right tibia and parenchyma mass accompanied by osteogenesis (Figure $1 B$; b5). Meanwhile, the different quantitative and morphologic neoplastic osteoids were observed by HE staining. The neoplastic osteoid existed in all the tissues with the background of malignant sarcomatoid cells. The typical neoplastic osteoids were irregular in a lace-like, network, or filigree osteoid pattern, and atypical neoplastic osteoblast was observed in normal remnant bone (Figure $2 A$ ).

\section{Characterization of Wells cells from induced tibial tumors}

The P2 orthotopic tumors were harvested and digested into single-cell suspension and plated in the dishes. After 10 generations of dilution, the purity of osteosarcoma cells was close to $100 \%$ (Figure 2B; b1,b2). We identified the purified osteosarcoma cells with SSEA-4 by immunofluorescence (Figure 2B; b3,b4) and named the purified osteosarcoma 
A

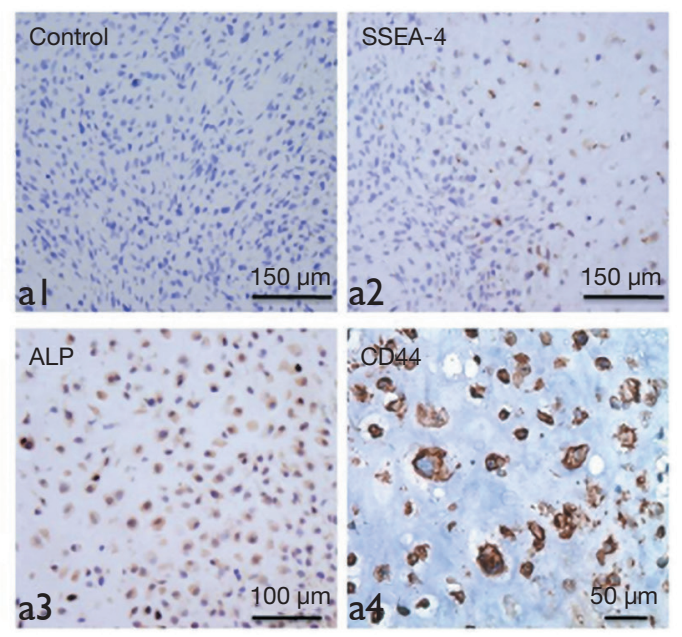

B
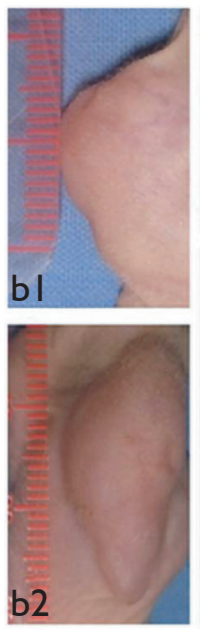

WJ0823
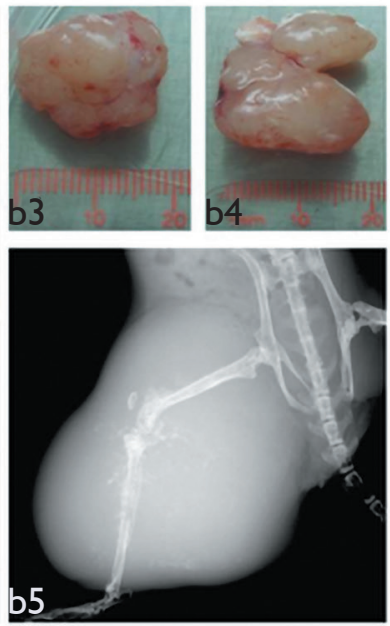

Figure 1 Establishment of an orthotopic model of osteosarcoma and tissue profiling. (A) Immunohistochemistry staining of the WJ0823 human osteosarcoma tissues; human osteosarcoma cells were pleomorphic and positive with SSEA-4 (a2), ALP (a3), and CD44 (a4). (B) NOD/SCID mice were inoculated minced human osteosarcoma tissue; the primary specimens generated P1 (b1,b3) and P2 (b2,b4) xenografts; X-ray demonstrated the destruction of the tibia (b5).
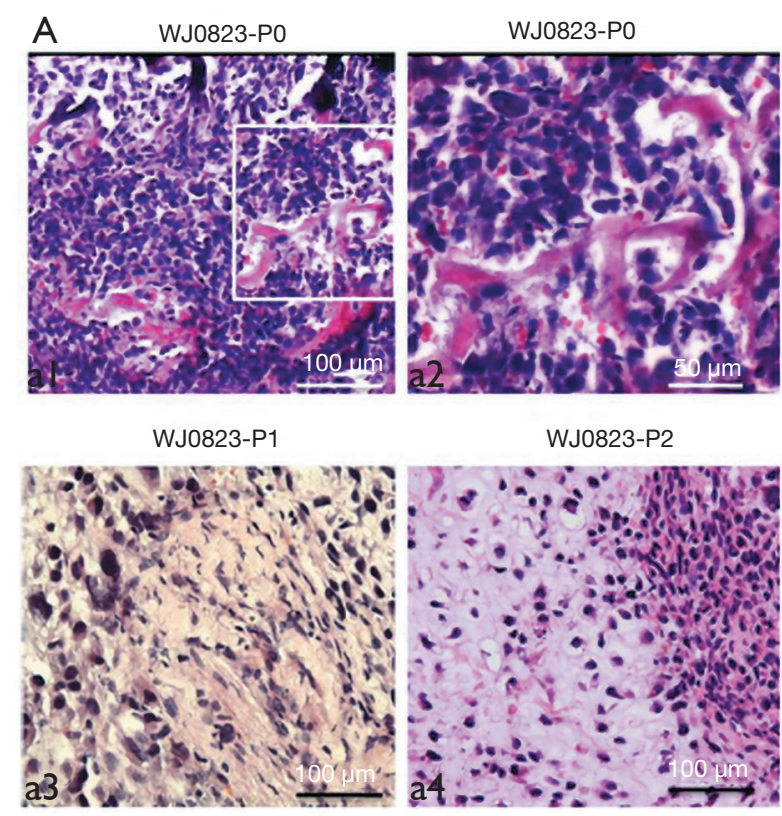
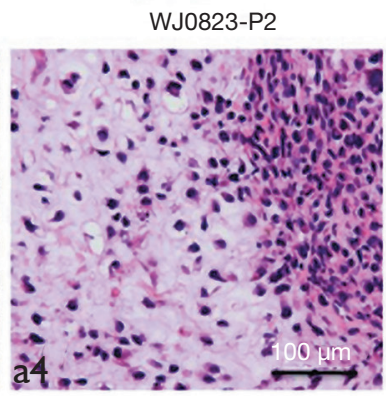

B
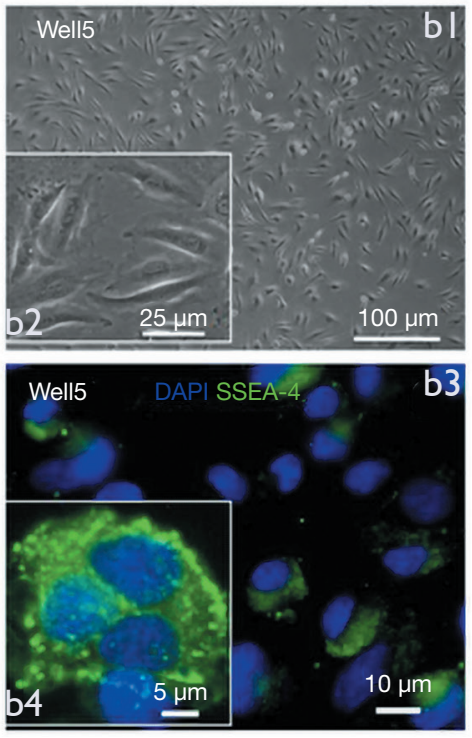

Figure $2 \mathrm{HE}$ staining of parent human osteosarcoma tissues and purification and characterization of Well5 cells from induced tibial tumors. (A) Parent (WJ0823-P0) (a2 is amplification of a1), passage 1 (WJ0823-P1) (a3) and passage 2 (WJ0823-P2). (a4) HE staining showed the formation of osteosarcoma. (B) The purity of osteosarcoma cells was close to $100 \%$ (b1,b2). Immunofluorescence showed the purification of osteosarcoma cells with SSEA-4 (b3,b4). 
A
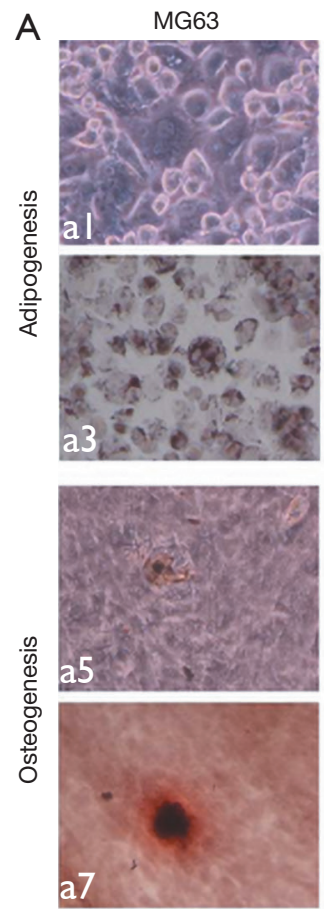

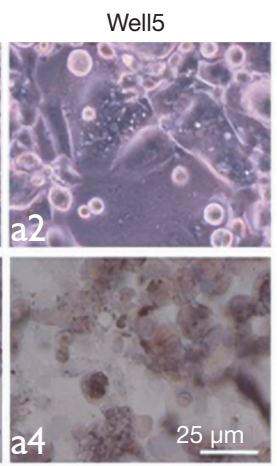

B
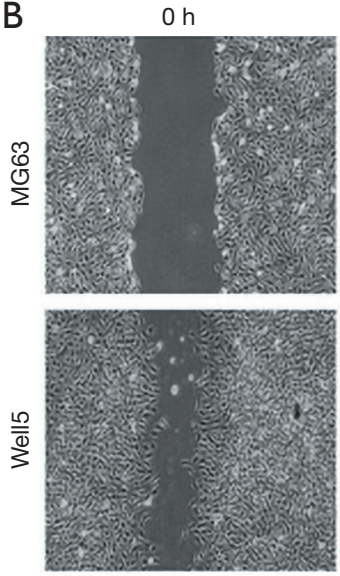
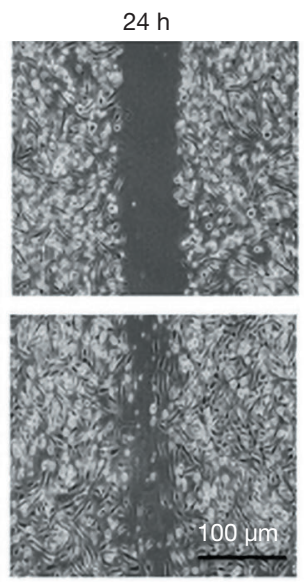

C

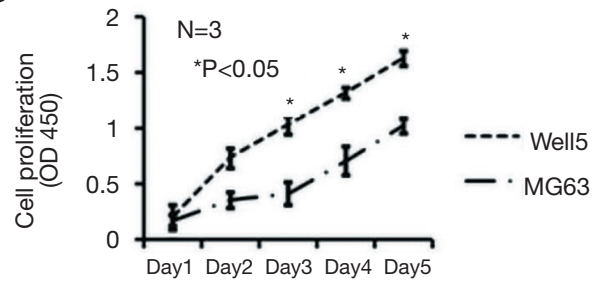

Figure 3 Adipogenic and osteogenic differentiation, migration, and proliferation of Well5 cells. In contrast to MG63 cells, we identified the adipogenesis, osteogenesis, migration, and proliferation ability of Well5 cells. (A) Well5 cells showed better abilities of adipogenesis (a2, a4) and osteogenesis (a6, a8) than MG63 (a1, a3 \& a5, a7); cell scratch assay indicated Well5 cells possessed stronger migration ability than MG63 (B); (C) we used the CCK8 to detect the proliferation of Well5 and MG63 cells. N=3/group, *, P<0.05. (A) DAB staining.

cells "Well5 cells".

\section{Adipogenesis, osteogenesis, migration, and proliferation of the Well5 cell line}

We further successfully induced Well5 cells to differentiate into adipogenic and osteogenic cells with conditions (Figure $3 A$ ) in vitro which indicated that Well5 cells had the ability of adipogenesis and osteogenesis. Cell scratch assay and C.CK8 assay indicated that Well5 cells obtained better capacity of migration (Figure $3 B$ ) and proliferation (Figure 3C) than the MG63 cell line.

\section{The orthotopic model of osteosarcoma and tumor growth at the primary site and spontaneous pulmonary metastases}

Next, the NOD/SCID mice were injected with Well5 cells to examine the ability of orthotopic tumor formation and spontaneous pulmonary metastasis development. As shown in Figure $4 A$ and $B$, tumors were observed in all the mice, being firmly involved into the tibia, and extended into the soft tissues around the bone. Moreover, pulmonary metastases were observed in all the mice. As shown in Figure $4 C$ and $D$, the pulmonary metastatic lesions extracted from Well5-cell-injected animals exhibited numerous surface metastases at as early as 6 weeks, which became more prominent by 8 weeks.

\section{Discussion}

By estimate, $\sim 10 \%$ to $20 \%$ of patients with osteosarcoma suffer from lung or bone metastasis at the time of diagnosis, and, in spite of progress achieved in recent clinical research, a large proportion of patients eventually die of pulmonary metastatic disease $(12,13)$. There is an urgent need to develop in vivo experimental systems, which will examine tumor growth and metastasis and the development of potential therapeutic agents.

Metastatic models in experimental animals are important to clarify the mechanism underlying tumor growth and metastasis. Tumor transplantation to rodents is the conventional way to establish metastasis models $(14,15)$. 

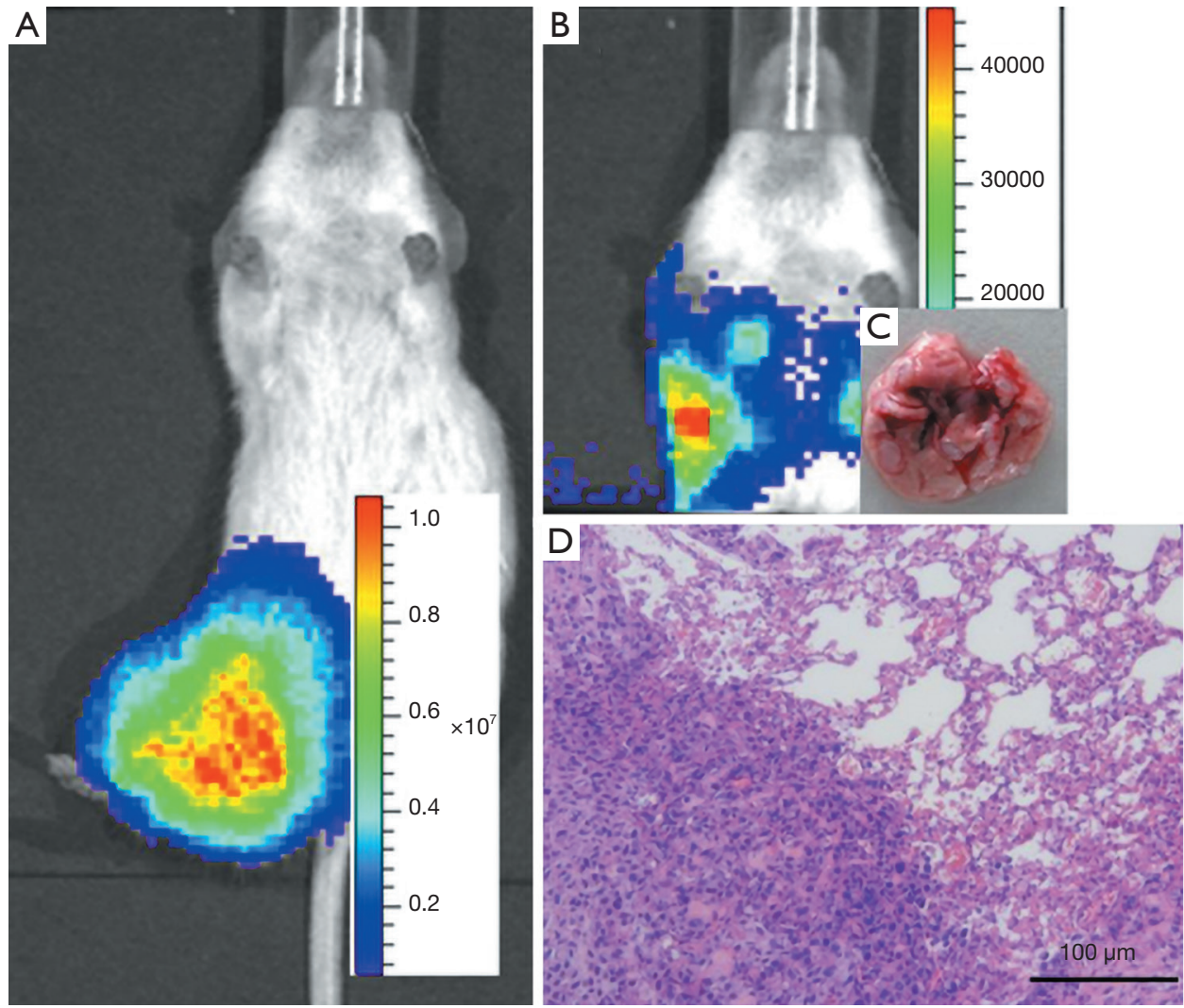

Figure 4 The destruction of the tibia and spontaneous pulmonary metastases. The destruction of the proximal tibia (A); the pulmonary metastasis image (B); histologic evaluation of the pulmonary metastases tissue (C); pulmonary metastasis histology at lower magnification (D). (D) HE staining.

While many of these models require administration of tumor cells intravenously or subcutaneously. The establishment of human osteosarcoma animal models in nude mice has been reported in previous studies $(16,17)$. Also, osteosarcoma was developed by injecting human tumor cells either intravenously or subcutaneously in some of these cases $(18,19)$. These animal models might not be clinically relevant because osteosarcoma cells are not spontaneously developed. Moreover, in these cases, tumor progression and metastases do not mimic the human clinical course.

In Jia et al.'s study, it was reported that a novel cell line named Saos-LM6 was isolated after 6 repeated cycles of tumor cells in xenograft mice (16), which was featured by pulmonary metastases. Su et al. established another new highly metastatic osteosarcoma cell line named MG63.2 from the parental MG63 cells (20). In the present study, we established a novel human osteosarcoma cell line, which was characterized by the development of spontaneous pulmonary metastasis after orthotopic implant of tumor cells. In the study, we found that Well5 cells could be orthotopically transplanted into NOD/SCID mice and that this resulted in local tumor invasion surrounding tissues and numerous spontaneous pulmonary metastases in a reproducible and quantifiable manner. Furthermore, Well5 cells presented a stronger ability for adipogenesis, osteogenesis, migration, and proliferation than MG63 cells. In this model, tumor progression and metastasis followed a human clinical scenario, which makes it a promising and reliable system for the evaluation strategy against osteosarcoma, especially against post-surgical micrometastatic disease.

In conclusion, our study established a novel osteosarcoma cell line namedWell5, which possessed osteoblast-like characteristics and might be a useful model for biological studies of osteosarcoma, and the development of novel strategies against osteosarcoma. 


\section{Acknowledgments}

Funding: This research was supported by the Chinese National Natural Science Foundation (No. 81172550).

\section{Footnote}

Conflicts of Interest: The authors have no conflicts of interest to declare.

Ethical Statement: All animal experiments were in accordance with the approved guidelines provided by the Laboratory Animal Resource Center of Shanghai Jiao Tong University School of Medicine. All the authors are accountable for all aspects of the work, and the questions related to the accuracy or integrity of any part of the work are appropriately investigated and resolved.

\section{References}

1. Yamanaka S, Arai H, Rino Y, et al. Pulmonary metastasis of metachronous triple cancer occurring in a young patient. Kyobu Geka 2008;61:541-4.

2. Whelan JS. Osteosarcoma. Eur J Cancer 1997;33:1611-8; discussion 1618-9.

3. Morello E, Martano M, Buracco P. Biology, diagnosis and treatment of canine appendicular osteosarcoma: similarities and differences with human osteosarcoma. Vet J 2011;189:268-77.

4. Postlethwaite C, Kumar J. An aggressive bone tumour: osteogenic osteomalacia. Clin Med (Lond) 2014;14:90.

5. Mankin HJ, Hornicek FJ, Rosenberg AE, et al. Survival data for 648 patients with osteosarcoma treated at one institution. Clin Orthop Relat Res 2004:286-91.

6. McQueen P, Ghaffar S, Guo Y, et al. The Wnt signaling pathway: implications for therapy in osteosarcoma. Expert Rev Anticancer Ther 2011;11:1223-32.

7. Picci P. Osteosarcoma (osteogenic sarcoma). Orphanet J Rare Dis 2007;2:6.

8. Yang J, Zhang W. New molecular insights into osteosarcoma targeted therapy. Curr Opin Oncol

Cite this article as: Yu P, Wen J, Wang J, Liang J, Shen Y, Zhang W. Establishment and characterization of a novel human osteosarcoma cell line for spontaneous pulmonary metastasis research in vivo. Ann Transl Med 2019;7(20):573. doi: 10.21037/ $\operatorname{atm} .2019 .09 .23$
2013;25:398-406.

9. Mc Auley G, Jagannathan J, O'Regan K, et al. Extraskeletal osteosarcoma: spectrum of imaging findings. AJR Am J Roentgenol 2012;198:W31-7.

10. Withrow SJ, Khanna C. Bridging the gap between experimental animals and humans in osteosarcoma. Cancer Treat Res 2009;152:439-46.

11. Berlin O, Samid D, Donthineni-Rao R, et al. Development of a novel spontaneous metastasis model of human osteosarcoma transplanted orthotopically into bone of athymic mice. Cancer Res 1993;53:4890-5.

12. Shiraishi K, Mori T, Ohba Y, et al. Three young osteosarcoma patients with small adenocarcinoma or atypical adenomatous hyperplasia of the lung. Ann Thorac Cardiovasc Surg 2010;16:358-61.

13. Endo-Munoz L, Cumming A, Rickwood D, et al. Loss of osteoclasts contributes to development of osteosarcoma pulmonary metastases. Cancer Res 2010;70:7063-72.

14. Rosol TJ, Tannehill-Gregg SH, LeRoy BE, et al. Animal models of bone metastasis. Cancer 2003;97:748-57.

15. Blouin S, Basle MF, Chappard D. Rat models of bone metastases. Clin Exp Metastasis 2005;22:605-14.

16. Jia SF, Worth LL, Kleinerman ES. A nude mouse model of human osteosarcoma lung metastases for evaluating new therapeutic strategies. Clin Exp Metastasis 1999;17:501-6.

17. Briccoli A, Rocca M, Ferrari S, et al. Surgery for lung metastases in Ewing's sarcoma of bone. Eur J Surg Oncol 2004;30:63-7.

18. Du L, Fan Q, Tu B, et al. Establishment and characterization of a new highly metastatic human osteosarcoma cell line derived from Saos2. Int J Clin Exp Pathol 2014;7:2871-82.

19. Liu X, Nielsen GP, Rosenberg AE, et al. Establishment and characterization of a novel chordoma cell line: $\mathrm{CH} 22$. J Orthop Res 2012;30:1666-73.

20. Su Y, Luo X, He BC, et al. Establishment and characterization of a new highly metastatic human osteosarcoma cell line. Clin Exp Metastasis 2009;26:599-610. 